\title{
TWO ELEMENTARY THEOREMS ON THE INTERPOLATION OF LINEAR OPERATORS ${ }^{1}$
}

\section{RICHARD O'NEIL}

Both theorems have to do with functions satisf ying Hölder conditions.

Definition. Let $T$ be an operator which takes functions whose domain is $n$-space into functions whose domain is a metric space. $T$ will be said to be of Hölder type $(\alpha, \beta)$ norm $N$ if for $g=T f$,

$$
|f(x)-f(x-h)| \leqq A|h|^{\alpha} \text { for all } x \text { and } h,
$$

implies

$$
|g(u)-g(v)| \leqq N A|u-v|^{\beta} \quad \text { for all } u \text { and } v .
$$

(Throughout this paper, when dealing with a metric space we shall denote the distance between $u$ and $v$ by $|u-v|$.)

Theorem 1. Suppose that $0 \leqq \alpha_{0} \leqq \alpha_{1} \leqq 1, \beta_{0} \geqq 0, \beta_{1} \geqq 0$ and that $T$ is a linear operator taking functions whose domain is $n$-space into functions whose domain is a metric space. If $T$ is simultaneously of Hölder type $\left(\alpha_{0}, \beta_{0}\right)$ norm $N_{0}$ and of Hölder type $\left(\alpha_{1}, \beta_{1}\right)$ norm $N_{1}$ and if $0 \leqq t \leqq 1$, then $T$ is of Hölder type $(\alpha, \beta)$ norm $N$ where

$$
\begin{aligned}
\alpha & =\alpha_{t}=\alpha_{0}(1-t)+\alpha_{1} t, \\
\beta & =\beta_{t}=\beta_{0}(1-t)+\beta_{1} t, \\
N & \leqq R_{n} N_{0}^{1-t} N_{1}^{t},
\end{aligned}
$$

and where $R_{n}$ depends only on the dimension of $n$-space.

Proof. Without loss of generality we may assume

$$
|f(x)-f(x-h)| \leqq|h|^{\alpha} .
$$

We first prove the theorem in case the domain of $f$ is the real line, that is when $n=1$.

For $r>0$, let

$$
K_{r}(s)= \begin{cases}1 / r-|s| / r^{2} & \text { if }|s|<r \\ 0 & \text { if }|s| \geqq r .\end{cases}
$$

Then

Presented to the Society, January 23, 1964 under the title Interpolation of operators for Lip spaces; received by the editors October 16, 1963.

1 This research was supported by the Air Force Office of Scientific Research. 


$$
\begin{aligned}
& \int K_{r}(s) d s=\int_{|\varepsilon|<r} K_{r}(s) d s=1, \\
& \int K_{r}^{\prime}(s) d s=0,
\end{aligned}
$$

and,

$$
\int\left|K_{r}^{\prime}(s)\right| d s=2 / r
$$

Let

$$
f_{r}(x)=\int f(x-s) K_{r}(s) d s=\int f(s) K_{r}(x-s) d s .
$$

Let $\epsilon_{r}(x)=f(x)-f_{r}(x), g=T f, g_{r}=T f_{r}$, and $\eta_{r}=T \epsilon_{r}$. Then $g=g_{r}+\eta_{r}$ by linearity of $T$.

$$
\begin{aligned}
f_{r}^{\prime}(x) & =\int f(s) K_{r}^{\prime}(x-s) d s=\int f(x-s) K_{r}^{\prime}(s) d s \\
& =\int(f(x-s)-f(x)) K_{r}^{\prime}(s) d s . \\
\left|f_{r}^{\prime}(x)\right| & \leqq \int_{|s|<r}|f(x-s)-f(x)|\left|K_{r}^{\prime}(s)\right| d s \leqq r^{\alpha}(2 / r)=2 r^{\alpha-1} .
\end{aligned}
$$

Case 1. $|h|<r$.

$$
\begin{aligned}
\left|f_{r}(x)-f_{r}(x-h)\right| & \leqq|h| \sup _{\nu}\left|f_{r}^{\prime}(y)\right| \leqq 2|h| r^{\alpha-1} \\
& =2|h|^{\alpha_{1}}|h|^{1-\alpha_{1} r^{\alpha-1}} \leqq 2|h|^{\alpha_{1} r^{\alpha-\alpha_{1}}} .
\end{aligned}
$$

Case 2. $|h| \geqq r$.

$$
\begin{aligned}
\left|f_{r}(x)-f_{r}(x-h)\right| & =\left|\int(f(x-s)-f(x-h-s)) K_{r}(s) d s\right| \\
& \leqq|h|^{\alpha}=|h|^{\alpha_{1}}|h|^{\alpha-\alpha_{1}} \leqq|h|^{\alpha_{1} \gamma^{\alpha-\alpha_{1}} .}
\end{aligned}
$$

In either case, $f_{r}$ satisfies a Hölder condition of order $\alpha_{1}$, indeed,

$$
\left|f_{r}(x)-f_{r}(x-h)\right| \leqq 2 r^{\alpha-\alpha_{1}}|h|^{\alpha_{1}} .
$$

Thus,

$$
\left|g_{r}(u)-g_{r}(v)\right| \leqq\left. N_{1} 2 r^{\sigma-\alpha_{1}}|u-v|\right|^{\beta_{1}}
$$




$$
\begin{aligned}
\epsilon_{r}(x)=f(x)-f_{r}(x) & =\int(f(x)-f(x-s)) K_{r}(s) d s . \\
\left|\epsilon_{r}(x)\right| & \leqq \int_{|s|<r}|s|{ }^{\alpha} K_{r}(s) d s \leqq r^{\alpha} .
\end{aligned}
$$

Case $1 .|h| \geqq r$.

$$
\left|\epsilon_{r}(x)-\epsilon_{r}(x-h)\right| \leqq 2 r^{\alpha} \leqq 2 r^{\alpha-\alpha_{0}}|h|^{\alpha_{0}} .
$$

Case 2. $|h|<r$.

$$
\begin{aligned}
\left|\epsilon_{r}(x)-\epsilon_{r}(x-h)\right| & \leqq|f(x)-f(x-h)|+\left|f_{r}(x)-f_{r}(x-h)\right| \\
& \leqq|h|^{\alpha}+|h|^{\alpha} \leqq 2|h|^{\alpha_{0}} r^{\alpha-\alpha_{0}} .
\end{aligned}
$$

Thus $\epsilon_{r}$ satisfies a Hölder condition of order $\alpha_{0}$. Therefore,

$$
\left|\eta_{r}(u)-\eta_{r}(v)\right| \leqq N_{0} 2 r^{\alpha-\alpha_{0}}|u-v| \beta_{0} .
$$

Thus, if we set $r=\left(N_{1}|u-v| \beta_{1}-\beta_{0} / N_{0}\right)^{1 /\left(\alpha_{1}-\alpha_{0}\right)}$,

$$
\begin{aligned}
|g(u)-g(v)| & \leqq\left|g_{r}(u)-g_{r}(v)\right|+\left|\eta_{r}(u)-\eta_{r}(v)\right| \\
& \leqq 2 N_{1} r^{\alpha-\alpha_{1}}|u-v|^{\beta_{1}}+2 N_{0} r^{\alpha-\alpha_{0}}|u-v|^{\beta_{0}} \\
& =4 N_{0}^{1-t} N_{1}^{t}|u-v|^{\beta} .
\end{aligned}
$$

This proves the theorem when the domain of $f$ is one dimensional. For $n>1$, the case $n=2$ is already sufficiently general to illustrate the proof. In this case we let

$$
K_{r}(s)= \begin{cases}3 / \pi r^{2}-3|s| / \pi r^{3} & \text { if }|s|<r, \\ 0 & \text { if }|s| \geqq r .\end{cases}
$$

For a given $h=\left(h_{1}, h_{2}\right) \neq 0$, let $\partial / \partial \theta$ denote directional differentiation in the direction $\theta=h /|h|$. Then $\int(\partial / \partial \theta) K_{r}(s) d s$ vanishes and $\int\left|(\partial / \partial \theta) K_{r}(s)\right| d s=O(1 / r)$. Thus,

$$
\begin{aligned}
\left|(\partial / \partial \theta) f_{r}(x)\right| & \leqq \int_{|s|<r}|f(x-s)-f(x)|\left|(\partial / \partial \theta) K_{r}(s)\right| d s \\
& \leqq r^{\alpha} O(1 / r)=O\left(r^{\alpha-1}\right) .
\end{aligned}
$$

Therefore, if $0<|h|<r, \theta=h /|h|$,

$$
\begin{aligned}
\left|f_{r}(x)-f_{r}(x-h)\right| & \leqq|h| \sup _{\nu}\left|(\partial / \partial \theta) f_{r}(y)\right|=|h| O\left(r^{\alpha-1}\right) \\
& =O\left(|h|^{\left.\alpha_{1} r^{\alpha-\alpha_{1}}\right) .}\right.
\end{aligned}
$$

The rest of the proof goes through as before. 
Definition. An operator $T$ is said to take $L^{p}$ into Lip $\alpha$ with norm $N$ if for $g=T f$,

$$
|g(u)-g(v)| \leqq N\|f\|_{p}|u-v|^{\alpha} \text { for all } u \text { and } v .
$$

If $f$ is a measurable function and $y>0$, let

$$
m(f, y)=m(|f|, y)=\text { measure of }\{x:|f(x)|>y\} .
$$

It is easily shown that

$$
\int|f(x)| d x=\int_{0}^{\infty} m(f, y) d y .
$$

Furthermore, for $p>0$,

$$
\begin{aligned}
m\left(|f|^{p}, y\right) & =\operatorname{meas}\left\{x:|f(x)|^{p}>y\right\} \\
& =\operatorname{meas}\left\{x:|f(x)|>y^{1 / p}\right\}=m\left(f, y^{1 / p}\right) .
\end{aligned}
$$

Thus,

$$
\begin{aligned}
\left(\|f\|_{p}\right)^{p} & =\int|f(x)|^{p} d x=\int_{0}^{\infty} m\left(|f|^{p}, v\right) d v \\
& =\int_{0}^{\infty} m\left(f, v^{1 / p}\right) d v=p \int_{0}^{\infty} m(f, y) y^{p-1} d y .
\end{aligned}
$$

Given $k \geqq 0$, let

$$
f_{k}(x)= \begin{cases}f(x) & \text { if }|f(x)| \leqq k \\ k \operatorname{sgn} f(x) & \text { if }|f(x)|>k\end{cases}
$$

and let

$$
f^{k}(x)=f(x)-f_{k}(x) .
$$

THEOREM 2. Suppose that $0<p_{0} \leqq p_{1} \leqq \infty, \alpha_{0} \geqq 0, \alpha_{1} \geqq 0$, and that $T$ is a linear operator taking measurable functions on a measure space into functions whose domain is a metric space. If $T$ simultaneously takes $L^{p_{0}}$ into Lip $\alpha_{0}$ with norm $N_{0}$ and $L^{p_{1}}$ into Lip $\alpha_{1}$ with norm $N_{1}$ and if $0 \leqq t \leqq 1$, then $T$ takes $L^{p}$ into Lip $\alpha$ with norm $N$ where

$$
\begin{aligned}
1 / p & =1 / p_{t}=(1-t) / p_{0}+t / p_{1}, \\
\alpha & =\alpha_{t}=(1-t) \alpha_{0}+t \alpha_{1}, \\
N & \leqq N_{0}^{1-t} N_{1}^{t} /(1-t)^{1-t} t^{t} \leqq 2 N_{0}^{1-t} N_{1}^{t} .
\end{aligned}
$$

(It is to be remarked that $1 /(1-t)^{1-t} t^{t}$ tends to 1 as $t$ tends to 0 or 1.) 
Proof. Suppose, without loss of generality, that $\|f\|_{p}=1$. Fix $k \geqq 0$, then $f=f^{k}+f_{k}$. Let $g=T f, g_{0}=T f^{k}$ and $g_{1}=T f_{k}$, then $g=g_{0}+g_{1}$ by linearity of $T$.

$$
\begin{aligned}
\left(\left\|f^{k}\right\|_{p_{0}}\right)^{p_{0}} & =p_{0} \int_{0}^{\infty} y^{p_{0}-1} m\left(f^{k}, y\right) d y=p_{0} \int_{0}^{\infty} y^{p_{0}-1} m(f, y+k) d y \\
& =p_{0} \int_{k}^{\infty}(z-k)^{p_{0}-1} m(f, z) d z \leqq p_{0} \int_{k}^{\infty} z^{p_{0}-1} m(f, z) d z \\
& \leqq p_{0} k^{p_{0}-p} \int_{k}^{\infty} z^{p-1} m(f, z) d z \leqq\left(p_{0} k^{p_{0}-p} / p\right)\left(\|f\|_{p}\right)^{p} \\
& =p_{0} k^{p_{0}-p} / p .
\end{aligned}
$$

Thus $\left\|f^{k}\right\|_{p_{0}} \leqq\left(p_{0} / p\right)^{1 / p_{0} k^{1-p / p_{0}}}$; since $T$ takes $L^{p_{0}}$ into Lip $\alpha_{0}$ with norm $N_{0}$,

$$
\begin{aligned}
\left|g_{0}(u)-g_{0}(v)\right| & \leqq N_{0}\left(p_{0} / p\right)^{1 / p_{0}} k^{1-p / p_{0}}|u-v|^{\alpha_{0}} \\
\left(\left\|f_{k} \mid\right\|_{p_{1}}\right)^{p_{1}} & =p_{1} \int_{0}^{\infty} y^{p_{1}-1} m\left(f_{k}, y\right) d y=p_{1} \int_{0}^{k} y^{p_{1}-1} m(f, y) d y \\
& \leqq p_{1} k^{p_{1}-p} \int_{0}^{k} y^{p-1} m(f, y) d y \leqq p_{1} k^{p_{1}-p} / p .
\end{aligned}
$$

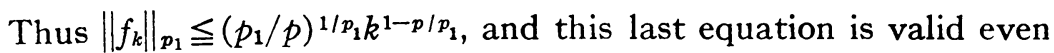
if $p_{1}=\infty$.

$$
\left|g_{1}(u)-g_{1}(v)\right| \leqq N_{1}\left(p_{1} / p\right)^{1 / p_{1}} k^{1-p / p_{1}}|u-v|^{\alpha_{1}} .
$$

If we set $A=1 / p_{0}-1 / p_{1}$, then $1 / p-1 / p_{1}=A(1-t)$ and $1 / p_{0}-1 / p=A t$

Thus, if we let

$$
\begin{aligned}
& k^{p \Lambda}=(t / 1-t)\left(p_{0} / p\right)^{1 / p_{0}}\left(p_{1} / p\right)^{-1 / p_{1}}\left(N_{0} / N_{1}\right)|u-v| \alpha_{0}-\alpha_{1}, \\
& |g(u)-g(v)| \leqq\left|g_{0}(u)-g_{0}(v)\right|+\left|g_{1}(u)-g_{1}(v)\right| \\
& =N_{0}\left(p_{1} / p\right)^{1 / p_{0} k^{-p A t}}|u-v| \alpha_{0} \\
& +N_{1}\left(p_{1} / p\right)^{1 / p_{1} k^{p A(1-t)}}|u-v|^{\alpha_{1}} \\
& =N_{0}^{1-t} N_{1}^{t}\left(1 / t^{t}(1-t)^{1-t}\right)\left(p_{0} / p\right)^{1-t / p_{0}}\left(p_{1} / p\right)^{t / p_{1}}|u-v|^{\alpha} \text {. }
\end{aligned}
$$

Let

$$
B=\left(p_{0} / p\right)^{(1-t) / p_{0}}\left(p_{1} / p\right)^{t / p_{1}}
$$

then 
$\log B=(1 / p) \log (1 / p)-(1-t)\left(1 / p_{0}\right) \log \left(1 / p_{0}\right)-t\left(1 / p_{1}\right) \log \left(1 / p_{1}\right)$.

But $x \log x$ is a convex function of $x \geqq 0$, so that

$$
(1 / p) \log (1 / p) \leqq(1-t)\left(1 / p_{0}\right) \log \left(1 / p_{0}\right)+t\left(1 / p_{1}\right) \log \left(1 / p_{1}\right) .
$$

Thus $\log B \leqq 0, B \leqq 1$ and the theorem is established.

REMARK. It is possible to strengthen the result of Theorem 2. We shall say that a measurable function $f$ belongs to weak $L^{p}$ if there exists a number $A$ such that for all $y>0$,

$$
m(f, y) \leqq(A / y)^{p} .
$$

If $f \in L^{p}$ then $f$ belongs to weak $L^{p}$, since

$$
\begin{aligned}
\left(\|f\|_{p}\right)^{p} & =p \int_{0}^{\infty} m(f, u) u^{p-1} d u \geqq p \int_{0}^{y} m(f, u) u^{p-1} d u \\
& \geqq p m(f, y) \int_{0}^{y} u^{p-1} d u=m(f, y) y^{p} .
\end{aligned}
$$

Thus,

$$
m(f, y) \leqq\left(\|f\|_{p} / y\right)^{p} .
$$

We shall say that a function $f \in \operatorname{Lip} \alpha$ if for all $u$ and $v$,

$$
|f(u)-f(v)| \leqq A|u-v|^{\alpha} .
$$

We shall say $f \in \operatorname{Lip} \alpha$ if

$$
|f(u)-f(v)|=o\left(|u-v|^{\alpha}\right)
$$

as $|u-v|$ tends to zero or infinity.

$1^{\circ}$. Under the hypotheses of Theorem $2, T$ takes weak $L^{p}$ into Lip $\alpha$ if $p_{0}<p<p_{1}$.

$2^{\circ}$. Under the hypotheses of Theorem $2, T$ takes $L^{p}$ into $\operatorname{Lip} \alpha$ if $p_{0}<p<p_{1}$.

To prove $1^{\circ}$, we suppose that $m(f, y) \leqq 1 / y^{p}$. Then

$$
\begin{aligned}
\left(\left\|f^{k}\right\|_{p_{0}}\right)^{p_{0}} & \leqq p_{0} \int_{k}^{\infty} z^{p_{0}-1} m(f, z) d z \leqq\left(p_{0} / p-p_{\theta}\right) k^{p_{0}-p}, \\
\left\|f^{k}\right\|_{p_{0}} & \leqq\left(p_{0} / p-p_{0}\right)^{1 / p_{0}} k^{1-p / p_{0}} .
\end{aligned}
$$

Similarly,

$$
\left\|f_{k}\right\|_{p_{1}} \leqq\left(p_{1} / p_{1}-p\right)^{1 / p_{1} k^{1-p / p_{1}}}
$$

Thus, if we let 


$$
\begin{gathered}
k^{p A}=\left(N_{0} / N_{1}\right)|u-v|^{\alpha_{0}-\alpha_{1}}, \\
|g(u)-g(v)| \leqq N_{0}^{1-t} N_{1}^{t}|u-v|^{\alpha}\left\{\left(p_{0} / p-p_{0}\right)^{1 / p_{0}}+\left(p_{1} / p_{1}-p\right)^{1 / p_{1}}\right\} .
\end{gathered}
$$

To prove $2^{\circ}$, we observe that

$$
\left(\|f\|_{p}\right)^{p}=\int_{0}^{\infty} m\left(f, v^{1 / p}\right) d v .
$$

Since $m\left(f, v^{1 / p}\right)$ is a monotone function of $v$, the finiteness of the integral implies

$$
m\left(f, v^{1 / p}\right)=o(1 / v) \text { as } v \text { tends to zero or infinity. }
$$

Thus,

$$
m(f, y)=o\left(1 / y^{p}\right) \text { as } y \text { tends to zero or infinity. }
$$

Therefore,

$$
\left\|f^{k}\right\|_{p_{0}}=o\left(k^{1-p / p_{0}}\right) \text { as } k \text { tends to zero or infinity, }
$$

and

$$
\left\|f_{k}\right\|_{p_{1}}=o\left(k^{1-p / p_{1}}\right) \text { as } k \text { tends to zero or infinity. }
$$

Again we may let

$$
k^{p A}=\left(N_{0} / N_{1}\right)|u-v| \alpha_{0}-\alpha_{1} .
$$

Thus,

$$
|g(u)-g(v)|=o\left(|u-v|^{\alpha}\right) \text {. }
$$

\section{REFERENCES}

1. R. Fiorenza, Theoremi di interpolazione per transformazioni tra spazi di funzioni con derivate hölderiane, Ricerche Mat. 10 (1961), 282-298.

2. - Ulteriori risultati sulla interpolasione tra spazi di funzioni con derivate hölderiane, Richerche Mat. 11 (1962), 224-253.

RICE UNIVERSITY 